\title{
Experimentelle Einzelfallstudien: eine Alternative zu randomisierten kontrollierten Studien?
}

\author{
Agnes von Wyl \& Aureliano Crameri \\ Psychotherapie-Wissenschaft 9 (2) 70-71 2019 \\ www.psychotherapie-wissenschaft.info \\ CC BY-NC-ND \\ https://doi.org/10.30820/1664-9583-2019-2-70
}

\begin{abstract}
Zusammenfassung: Experimentelle Einzelfallstudien werden immer häufiger auch in der Psychotherapieforschung eingesetzt. Es stellt sich die Frage, ob sie eine Alternative zu randomisierten kontrollierten Studien (randomized controlled trials; RCTs) darstellen. Sie könnten eine Möglichkeit für psychotherapeutische Institute sein, mit weniger Fällen als es eine RCT verlangt, den Anspruch der Forschung auf eine Evidenzbasierung ihres psychotherapeutischen Ansatzes zu erfüllen.
\end{abstract}

Schlüsselwörter: Evidenzbasierung, Experimentelle Einzelfallstudie, RCT

Experimentelle Einzelfallstudien werden immer häufiger auch in der Psychotherapieforschung eingesetzt (s. z. B. den Artikel von Herrera et al. in diesem Heft). Für psychotherapeutische Ausbildungsinstitute stellt sich die Frage, ob sie eine Alternative zu randomisierten kontrollierten Studien (randomized controlled trials; RCTs) darstellen. Insbesondere ist zu beurteilen, welchen Stellenwert ihnen in der Forschung zur evidenzbasierten Psychotherapie zugestanden wird.

Experimentelle Einzelfallstudien werden von der APA als hilfreich zur Einschätzung von kausalen Zusammenhängen bei individuellen Therapieverläufen angeführt; neben weiteren Forschungsdesigns könnten sie damit einen wichtigen Beitrag zur evidence based practice beitragen. Für die Belegung des ursächlichen Zusammenhangs zwischen einer Intervention und dem Therapieergebnis führen sie RCT-Designs auf (APA, 2006). Bei Chambless und Ollendick (2001) gilt eine Serie von experimentellen Einzelfallstudien unter der Voraussetzung, dass dabei ein Therapiemanual bzw. eine eindeutige Beschreibung des Behandlungsansatzes eingesetzt wird, als äquivalent zu einer RCT.

Doch was beinhaltet eine experimentelle Einzelfallstudie? Als Experimente können sie kausale Zusammenhänge für den Einzelfall abbilden. Dabei werden die Effekte unter mindestens zwei unterschiedlichen Bedingungen (z. B. Baseline und Intervention) auf ein Therapieergebnis hin miteinander verglichen. Anhand der Abbildungen 1 und 2 sollen zwei mögliche Schemata eines Einzelfall-Designs erläutert werden. In beiden Schemata werden jeweils die Wirkung einer Intervention (B) sowie die jeweilige Baseline (A) vor der Therapie dargestellt.

Im ersten Schema (Abb. 1) wird acht Tage lang täglich das Ausmass der Symptomatik erfasst. Ab dem neunten Tag erfolgt über zehn Tage hinweg die Intervention. Auch hier wird täglich das Ausmass der Symptomatik erhoben.

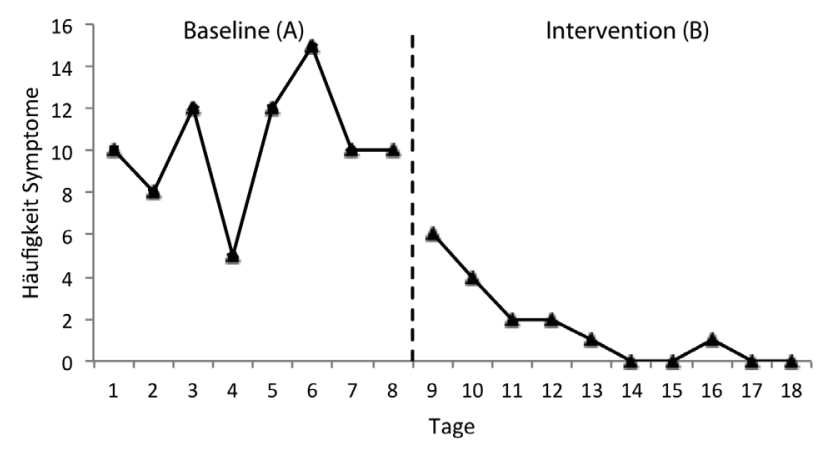

Abb. 1: Einfaches Baseline-Design

Im zweiten Schema (Abb. 2) gibt es zwei Vergleichsphasen zur Intervention, nämlich wiederum die Zeit vor der Therapie, aber zusätzlich noch die Zeit danach. Im von Herrera et al. (i.d. Heft) vorgestellten Beispiel wurde dieses zweite Schema angewendet; die Therapien dauerten zwischen acht und 40 Sitzungen.

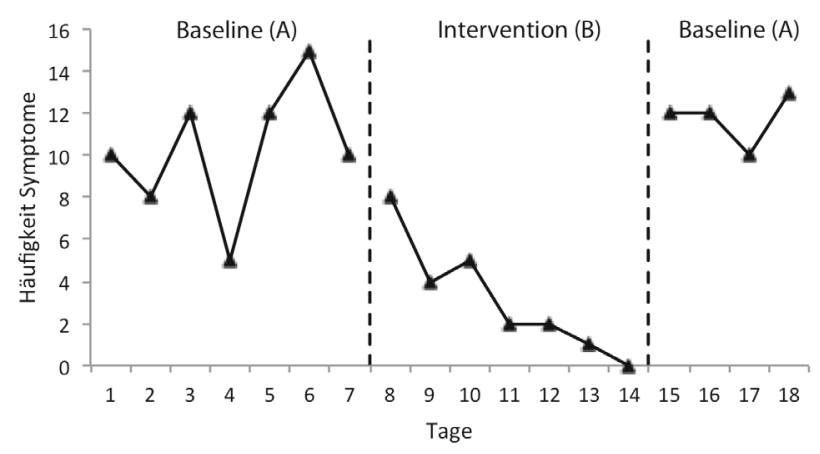

Abb. 2: Umkehr-Design

Als eine mögliche Variation soll folgendes Bespiel vorgestellt werden: Als Intervention könnte zum Beispiel auch 
eine bestimmte körpertherapeutische Übung definiert werden, die ab der neunten Sitzung im Rahmen einer Psychotherapie eingesetzt wird. Die Psychotherapie ohne diese körpertherapeutische Übung wäre dann die Baseline. Mit diesem Design liesse sich die Wirkung einer einzelnen Technik auf das Therapieergebnis einschätzen. Zusätzlich könnte man dieses Design mit Kontrollfällen vergleichen, das heisst mit Therapien, in denen nur die Therapie ohne die Zusatzintervention angewandt wird.

Als wichtige Voraussetzung für experimentelle Einzelfallstudien ist ausserdem zu berücksichtigen, dass kontinuierliche Erhebungen zur Symptomatik oder zu anderen interessierenden Indikatoren bzw. Faktoren als Grundlage der Auswertung nötig sind. Dies gilt auch für die Basiserhebung (s. a. Kazdin, 2016). Es müssen also für alle drei Phasen über einen bestimmten Zeitraum hinweg Daten erhoben werden, das heisst eine einmalige Fragebogenerhebung vor Beginn der Therapie reicht nicht aus. Für das genauere Vorgehen bei experimentellen Einzelfallstudien möchten wir auf weiterführende Literatur verweisen (z.B. ebd. sowie Manolov et al., 2014). Über die Schwierigkeiten, die die Durchführung einer Einzelfallstudie beinhalten kann, findet man Ausführungen bei Michiels und Onghena (2018).

Experimentelle Einzelfallstudien können also eine Möglichkeit für psychotherapeutische Institute sein, mit weniger Fällen als eine RCT, den Anspruch der Forschung auf eine Evidenzbasierung ihres psychotherapeutischen Ansatzes zu erfüllen. Allerdings muss man sich bewusst sein, dass «experimentell» auch bedeutet, die Therapien nach bestimmten, vorher definierten Kriterien durchzuführen. Diesbezüglich unterscheidet sich die experimentelle Einzelfallstudie nicht von einer RCT. Bei einer RCT erhalten alle Probanden der einen Gruppe eine bestimmte Intervention und alle Probanden der anderen Gruppe eine andere. Bei einer experimentellen Einzelfallstudie erhält ein/e einzelne/r PatientIn streng genommen zwei unterschiedliche Interventionen bzw. wird unterschiedlichen Konditionen unterzogen, nämlich «keine Intervention» im Vergleich zu «Intervention».

\section{Literatur}

APA Presidential Task Force on Evidence-Based Practice (2006). Evidence-based practice in psychology. American Psychologist, 61, 271-285. https://doi.org/10.1037/0003-066X.61.4.271

Chambless, D.L. \& Ollendick, T.H. (2001). Empirically supported psychological interventions: controversies and evidence. Annu Rev Psychol, 52, 685-716. https://doi.org/0.1146/annurev.psych.52.1. $68552 / 1 / 685$
Kazdin, A.E. (2016). Single-case experimental research designs. In A.E. Kazdin (Hrsg.), Methodological issues and strategies in clinical research (S. 459-483). Washington, D. C.: APA. https://doi. org/10.1037/14805-029

Manolov, R., Gast, D. L., Perdices, M. \& Evans, J.J. (2014). Single-case experimental designs: reflections on conduct and analysis. Neuropsychol Rehabil, 24, 634-660. https://doi.org/10.1080/0960 2011.2014.903199

Michiels, B. \& Onghena, P. (2018). Randomized single-case AB phase designs: Prospects and pitfalls. Behavior Research Methods, 51, 1-23. https://doi.org/10.3758/s13428-018-1084-x

\section{Experimental case studies: an alternative to randomized controlled studies?}

Abstract: Experimental case studies are increasingly used also in psychotherapy research. The question arises whether they represent an alternative to randomized controlled trials (RCTs). They could be a possibility for psychotherapeutic institutes to meet the requirements of research to base their psychotherapeutic approaches on evidence, with fewer cases than an RCT requires.

Key words: evidence-based, experimental case study, RCT

\section{Studi single-case sperimentali: un'alternativa agli studi controllati randomizzati?}

Riassunto: Gli studi single-case sperimentali sono sempre più utilizzati nella ricerca in psicoterapia. Ci si chiede se rappresentino un'alternativa agli studi controllati randomizzati controllati (randomized controlled trials; RCT). Potrebbero essere una possibilità per gli istituti psicoterapeutici, con un numero di casi inferiore a quello richiesto da un RCT, per soddisfare l'esigenza della ricerca sull'evidence-basing del loro approccio psicoterapeutico.

Parole chiave: evidence-basing, studi single-case sperimentali, RCT

\section{Die Autorlnnen}

Agnes von Wyl, Prof. Dr., ist Leiterin der Fachgruppe Klinische Psychologie und Gesundheitspsychologie an der Zürcher Hochschule für Angewandte Wissenschaften, Departement Psychologie.

Aureliano Crameri, Dr., ist Dozent für Forschungsmethoden und wissenschaftlicher Mitarbeiter an der Zürcher Hochschule für Angewandte Wissenschaften, Departement Psychologie.

\section{Kontakt}

E-Mail: agnes.vonwyl@zhaw.ch

E-Mail: aureliano.crameri@zhaw.ch 